\title{
BONE
}

\section{Ronacaleret in osteoporosis}

Ronacaleret therapy increases trabecular volumetric BMD (vBMD) but reduces cortical vBMD in postmenopausal women, report researchers involved in the GlaxoSmithKline-funded clinical trial of this calcium-sensing receptor antagonist.

The trial included 569 postmenopausal women with low BMD. Patients were assigned either to open-label teriparatide $(20 \mu \mathrm{g})$ once daily or randomly assigned in a double-blinded fashion to different doses of ronacaleret $(100 \mathrm{mg}, 200 \mathrm{mg}$, $300 \mathrm{mg}$ or $400 \mathrm{mg}$ ) once daily, alendronate (70 mg) once weekly, or placebo. The follow-up period was 12 months. However, a 6-month analysis, whose results were published in August, showed that the increases in BMD, measured by dual-energy X-ray absorptiometry, achieved with ronecaleret were lower than those with teriparatide or alendronate, and treatment was phased out.

"It was important in the context of this new treatment to determine whether the effects were comparable, weaker or stronger in the cortical or trabecular bone," explains Harry Genant, one of the researchers. "The former is perhaps more important in reducing hip fractures, while the latter is more important in reducing spine fractures." Therefore, a substudy was undertaken on 314 of the women, in whom vBMD at the hip and spine was assessed by non-invasive quantitative CT.

Ronacaleret increased spine integral and trabecular vBMD from baseline. However, at the proximal femur small decreases in integral vBMD occurred with ronacaleret, whereas vBMD increased at this site in the alendronate and teriparatide groups.

"The treatment would likely not reduce hip fractures, the most important osteoporotic fractures," comments Genant. "In clinical practice this form of anabolic treatment will not be utilizable, at least in the form examined in this study."

\section{Rosanne Diaz}

Original article Fitzpatrick, A. et al. Ronacaleret, a calciumsensing receptor antagonist, increases trabecular but not cortical bone in postmenopausal women. J. Bone Miner. Res. doi:10.1002/jbmr.554 